\title{
Desafios na Gerência de Estacionamentos por Imagem
}

\author{
Paulo R. L. de Almeida, Eduardo C. de Almeida \\ Universidade Federal do Paraná (UFPR), Curitiba, Brasil
}

\begin{abstract}
Resumo. Com o aumento na população urbana, as cidades tem grande necessidade de utilizar métodos apoiados por técnicas de Inteligência Artificial para melhorar a mobilidade urbana em trânsitos cada vez mais congestionados pelo crescente número de veículos em circulação. Estudos demonstram que o problema de congestionamento de trânsito é agravado em até $30 \%$ por veículos procurando vagas de estacionamento. Neste artigo sumarizamos problemas em aberto na gerência de vagas de estacionamento por imagem e listamos nossos esforços de pesquisa combinando técnicas de aprendizado de máquina, edge computing e processamento de imagens.
\end{abstract}

\section{Introdução}

De acordo com as Nações Unidas, atualmente cerca de $55 \%$ da população mundial vive em áreas urbanas, sendo que esse número deve chegar a 66\% em 2050. No Brasil, estimase que $87 \%$ da população vive em áreas urbanas, e esse número deve ultrapassar os $90 \%$ em 2050. Vale ainda ressaltar que das 33 megacidades $^{1}$ do mundo, duas estão localizadas no Brasil [DESA 2019]: São Paulo e Rio de Janeiro.

A grande e ainda crescente população em áreas densamente povoadas cria desafios em diversas áreas, como na dos transportes, energia, e segurança. Nesse âmbito, as cidades inteligentes (ou cidades inteligentes sustentáveis - [Ahvenniemi et al. 2017]) utilizam recursos de hardware e software para integrar e analisar os dados de seus componentes de infraestrutura, utilizando-os mais eficientemente [Ahvenniemi et al. 2017, Letaifa 2015].

No contexto dos transportes, otimizações nos sistemas de temporização de semáforos [Li et al. 2017, Djahel et al. 2013], otimização do tráfego para serviços de emergência [Djahel et al. 2013], predição das condições de tráfego [Nagy and Simon 2018] e a gerência de vagas de estacionamento [de Almeida et al. 2015, Amato et al. 2017, Padmasiri et al. 2020] podem levar a diversos benefícios, como a redução de congestionamentos, redução do consumo de energia (e.g., combustíveis fósseis ou eletricidade), menor poluição, e um aumento na qualidade de vida dos habitantes.

Em [Koumetio Tekouabou et al. 2020] é discutido que os congestionamentos são agravados em até $30 \%$ por motoristas em busca de vagas. Assim, este trabalho foca nos problemas relativos a gerência de vagas de estacionamento por imagem em cidades inteligentes que ainda estão em aberto. Apesar de já existirem métodos para a classificação de vagas de estacionamento (entre vazias e ocupadas), há uma carência de métodos capazes de atender diversos requisitos, como: segmentar automaticamente as vagas; executar sem amostras de treinamento do estacionamento onde o sistema será implantado; utilizar

\footnotetext{
${ }^{1}$ De acordo com as Nações Unidas, uma megacidade possui mais de 10 milhões de hab. [DESA 2019]
} 
tecnologias emergentes como edge computing; e integrar os dados processados com planejadores de rotas. Além da carência dos métodos em si, há também a falta de bases de dados públicas para a criação e teste desses métodos.

O objetivo desse trabalho é apontar problemas relativos a gerência de vagas de estacionamento por imagem e chamar a atenção da comunidade científica para resolvêlos. A opção pela gerência através de imagem (sensores do tipo câmera) se dá pela simplicidade de instalação desse tipo de sistema, já que uma única câmera pode monitorar uma área ampla (exemplo na Figura 2). Além disso, tanto pátios de estacionamento como vias públicas comumente já contam com câmeras previamente instaladas (e.g., para segurança e monitoramento) que podem ser usadas para essa tarefa [de Almeida et al. 2015, Amato et al. 2017].

Para um melhor entendimento dos problemas, na Seção 2 é apresentado um breve estado da arte. Na seção 3 são listados os problemas que consideramos como abertos, sendo que para cada problema é apresentada uma pergunta de pesquisa principal. Atualmente, alguns problemas estão sendo tratados em nossos projetos em andamento, que são brevemente descritos na Seção 4. Finalmente, as conclusões são apresentadas na Seção 5.

\section{Estado da Arte}

A literatura de sistemas de gerência de vagas de estacionamento por imagem divide-se em métodos de classificação das vagas e métodos de segmentação de vagas. A classificação das vagas pode ser caracterizada como o processo de se inferir se determinada imagem (previamente recortada como na Figura 1) contendo exatamente uma vaga de estacionamento encontra-se ocupada ou vazia. Este é um problema amplamente estudado, sendo que os métodos propostos comumente se baseiam na extração de características para utilização posterior de classificadores [de Almeida et al. 2015, Ahrnbom et al. 2016, Bohush et al. 2018, Vítek and Melničuk 2018, Varghese and Sreelekha 2019], ou na utilização de redes neurais convolucionais [Amato et al. 2017, Nurullayev and Lee 2019, Jensen et al. 2017, Nieto et al. 2018, Zhang et al. 2019, Chen et al. 2020].

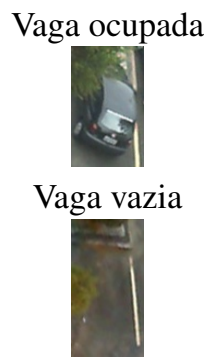

Figura Classificação².

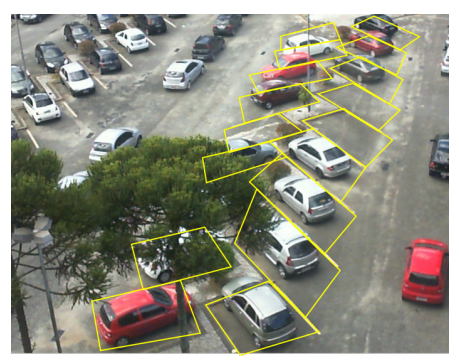

Figura 2. Segmentação de Vagas ${ }^{2}$.

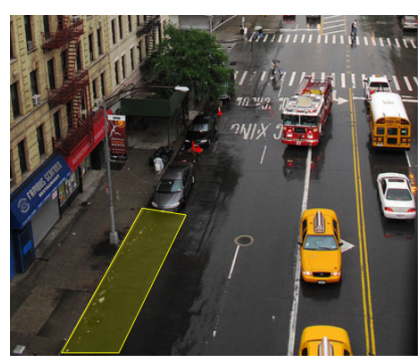

Figura 3. Detecção em vias públicas ${ }^{3}$.

Para os testes dos métodos de classificação de vagas individuais, podemos definir os seguintes cenários:

- Sem mudança: as imagens de treinamento e teste pertencem ao mesmo estacionamento e ângulo de câmera;

\footnotetext{
${ }^{2}$ Imagens da base de dados PKLot [de Almeida et al. 2015].

${ }^{3}$ Imagem da base de dados COCO [Lin et al. 2014].
} 
- Com mudança: as imagens de treinamento e teste pertencem a estacionamentos diferentes, e foram coletadas utilizando ângulos de câmera diferentes.

Esses cenários fazem parte do protocolo discutido em [de Almeida et al. 2015]. O cenário com mudança é obviamente mais desafiador, e pode levar ao desenvolvimento de sistemas capazes de serem instalados em qualquer área com vagas de estacionamento, sem a necessidade de ajustes finos. No entanto, como pode-se observar na Tabela 1, boa parte dos autores ignoram testes em cenários com mudança. Além disso, ao se analisar os resultados de acurácia média, pode-se identificar claramente que o problema de classificação torna-se mais desafiador quando o método não recebe amostras de treinamento do estacionamento de testes.

Tabela 1. Sumário das técnicas de classificação de vagas individuais ${ }^{4}$

\begin{tabular}{lrr}
\hline Autores & Acc. (\%) sem mudança & Acc. (\%) com mudança \\
\hline [de Almeida et al. 2015] & $99.3-99.6 \%$ & $84.2-89.8 \%$ \\
[Ahrnbom et al. 2016] & $0.999-1.000$ (AUC-ROC) & $0.940-0.996$ (AUC-ROC) \\
[Amato et al. 2017] & $90.1-98.1 \%$ & $92.7-98.3 \%$ \\
[Jensen et al. 2017] & $99.7-99.9 \%$ & $95.5-98.7 \%$ \\
[Bohush et al. 2018] & $99.7 \%$ & - \\
[Nieto et al. 2018] & 0.919 (AUC-PR) & - \\
[Vítek and Melničuk 2018] & $90.7-93.2 \%$ & $83-96 \%$ \\
[Nurullayev and Lee 2019] & $94.4-98.4 \%$ & - \\
[Varghese and Sreelekha 2019] & $91.1-99.7 \%$ & $81.7 \%$ \\
[Zhang et al. 2019] & $99.1-99.9 \%$ & - \\
[Chen et al. 2020] & $99 \%$ & - \\
\hline Média & $\mathbf{9 7 . 0 \%}$ & $\mathbf{9 0 . 1 \%}$ \\
\hline
\end{tabular}

A tarefa de classificação das vagas depende da demarcação das coordenadas de cada uma das vagas de estacionamento na imagem (veja um exemplo na Figura 2). As coordenadas são usadas para realizar a segmentação das vagas individuais, que serão classificadas. Já que a câmera fica em uma posição fixa, a demarcação manual das vagas é viável. No entanto, essa é uma tarefa tediosa que demanda tempo e que, apesar de aparentar ser simples, pode tornar-se especialmente difícil dependendo do ângulo da câmera e das condições do estacionamento. Além disso, mudanças de ângulo da câmera podem forçar a re-demarcação, tomando tempo humano e justificando a criação de sistemas capazes de realizar a segmentação de maneira automatizada.

Métodos para a segmentação automática de vagas de estacionamento utilizando técnicas clássicas de processamento de imagens, como transformações de perspectivas, binarizações e detectores de borda, são propostos em [Bohush et al. 2018, Zhang et al. 2019, Zhang and Du 2020]. Extratores de características são usados em [Vítek and Melničuk 2018] para detectar se blocos da imagem pertencem ou não a carros, e métodos baseados em redes neurais convolucionais são apresentados em [Agrawal and Urolagin 2020, Padmasiri et al. 2020, Hurst-Tarrab et al. 2020].

\footnotetext{
${ }^{4}$ Os resultados não são diretamente comparáveis. Diferentes autores utilizaram diferentes bases de dados e protocolos experimentais. Resultados apresentados como $X-Y$ indicam o melhor e o pior resultado obtido pelos autores. A média leva em consideração apenas resultados reportados como acurácias.
} 
Apesar de existirem métodos tratando da segmentação automática de vagas, os trabalhos são escassos e em sua grande maioria apresentam dados insuficientes sobre os métodos, experimentos e principalmente resultados. Dos trabalhos citados, apenas [Padmasiri et al. 2020, Hurst-Tarrab et al. 2020] apresentam resultados quantitativos.

\section{Problemas em Aberto}

\subsection{Classificação de vagas individuais com mudança de ambiente}

Como discutido no estado da arte (Seção 2), existem diversos métodos propostos para a classificação de vagas individuais com alta acurácia quando se considera que o método possui amostras de treinamento coletadas no mesmo ambiente onde serão realizados os testes. No entanto, quando o método é treinado em um estacionamento, e posto a prova em outro, as taxas de acertos caem significativamente.

A obtenção de amostras de treinamento do ambiente onde o sistema será instalado pode demandar muito tempo humano, e até mesmo inviabilizar a implantação do sistema. Sendo assim, métodos capazes de generalizar seus conhecimentos (e.g. aprender com bases de dados fixas e operar em qualquer ambiente de estacionamento sem a necessidade de amostras desses ambientes) ainda representam um problema em aberto.

Pergunta de pesquisa: como gerar um método de classificação de vagas individuais capaz de generalizar seu conhecimento para qualquer estacionamento?

\subsection{Segmentação Automatizada de Vagas}

São poucos os trabalhos que tratam da segmentação automatizada de vagas de estacionamento, como discutido na Seção 2. Além disso, os métodos propostos sofrem de diversos problemas graves, como a falta de métricas para se avaliar os resultados (somente dois métodos na Seção 2 reportam resultados quantitativos), falta de protocolos de testes padronizados, e falta de bases rotuladas especificamente para essa tarefa. Sendo assim, a segmentação automática de vagas de estacionamento é um problema ainda em aberto que, até o momento, foi abordado por poucos pesquisadores.

Pergunta de pesquisa: é possível detectar automaticamente as posições das vagas de estacionamento sem nenhuma informação a priori sobre o estacionamento em questão (e.g., sem amostras de treinamento ou parâmetros de câmera)?

\subsection{Criação de classificadores leves}

Segundo [Satyanarayanan 2017], a transmissão simultânea de diversos fluxos de vídeo em uma cidade inteligente pode facilmente sobrecarregar sua infraestrutura de rede. Com isso, faz-se necessário o desenvolvimento de métodos baseados em paradigmas emergentes de computação, como o edge computing [Satyanarayanan 2017]. Neste sentido, iniciativas de pesquisa devem levar em consideração restrições de custo computacional, de tráfego de rede de dados, e uso energético, para que pelo menos parte do processamento possa ser realizado diretamente onde o sensor se encontra (em um sistema embarcado), evitando assim o envio dos dados não tratados (e.g., fluxo de vídeo ou imagens) pela rede [Amato et al. 2017, Chen et al. 2020]. 
Muitos autores utilizam redes neurais convolucionais para, por exemplo, realizar a classificação de vagas individuais. Essas redes são interessantes pela sua relativa capacidade de generalização e pela desnecessidade de se selecionar extratores de características. No entanto, esses modelos robustos comumente demandam muitos recursos computacionais, o que dificulta suas utilizações em dispositivos embarcados, como smart cameras. Dessa forma, um problema ainda aberto é a modelagem de classificadores capazes de trabalhar com recursos computacionais e de energia reduzidos. Isso pode levar a soluções que podem ser implantadas diretamente em dispositivos embarcados, e contribuir com a redução de emissões de gases devido ao menor consumo de energia.

Pergunta de pesquisa: como criar classificadores leves, capazes de serem executados em dispositivos embarcados, e com acurácia e capacidade de generalização comparáveis ou levemente inferiores a classificadores "robustos"?

\subsection{Detecção de vagas de estacionamento em laterais de vias públicas}

Como as cidades comumente já possuem câmeras instaladas para monitorar muitas de suas vias de tráfego de veículos, um problema interessante que pode ser tratado é a detecção de vagas de estacionamento nas laterais das vias. Esse tipo de sistema pode gerar informações para que planejadores de rotas (e.g. Google Maps, Microsoft Bing, Strava) levem o motorista diretamente até a vaga mais próxima de seu destino. Esses sistemas podem ainda compor outros sistemas, como os de cobrança de estacionamento por tempo, ou sistemas de alerta que indicam veículos estacionados ilegalmente.

Esse problema é especialmente desafiador, já que é necessário detectar automaticamente as posições onde os carros podem parar, e classificar as posições entre vazias e ocupadas (uma combinação dos problemas das Seções 3.1 e 3.2). Detectar uma vaga vazia pode ser especialmente difícil, já que as vagas nas laterais das vias geralmente não são pré-determinadas. Um exemplo pode ser visualizado na Figura 3, onde o polígono amarelo demarca possíveis vagas vazias. Um sistema de gestão de estacionamento capaz de detectar vagas em vias públicas deve ser capaz de gerar esses polígonos, e indicar a quantidade de veículos que cada polígono pode comportar.

Pergunta de pesquisa: como identificar automaticamente vagas nas laterais das vias assumindo que as posições onde os carros estacionam não são fixas?

\subsection{Georreferenciamento}

Sistemas gerenciadores de vagas podem ser integrados a planejadores de rotas, levando os motoristas diretamente a vaga vazia mais próxima do seu destino. Para isso, é necessário que as imagens coletadas pelas câmeras sejam georreferenciadas. Isso é um desafio, já que não se pode esperar que tenhamos coordenadas precisas de latitude e longitude de uma quantidade razoável de pontos das imagens das câmeras. Dos trabalhos pesquisados, apenas [Hurst-Tarrab et al. 2020] tenta realizar a correspondência entre imagens de satélite e as imagens de câmeras de vigilância de estacionamentos. Isso indica que pesquisas nesse tópico ainda são necessárias. 
Pergunta de pesquisa: como georreferenciar as imagens de câmeras de estacionamento?

\subsection{Bases e protocolos}

Técnicas para o tratamento dos problemas listados nesta seção só podem ser desenvolvidas e testadas através do uso de bases de dados rotuladas, e através de protocolos experimentais padronizados, que devem ser seguidos a fim de tornar os resultados comparáveis e reprodutíveis. Existem bases rotuladas para a tarefa de classificação de vagas individuais em pátios de estacionamento, como a PKLot [de Almeida et al. 2015], CNRPark-EXT [Amato et al. 2017] e PLDs [Nieto et al. 2018]. Apesar de interessantes, algumas características importantes ainda precisam ser incorporadas nessas (ou em novas) bases, como a rotulação dos objetos através de máscaras de segmentação e o georreferenciamento das imagens. Isso pode auxiliar no desenvolvimento de métodos, principalmente para os problemas descritos nas Seções 3.2, 3.4 e 3.5. Novas bases contendo sequências de vídeo e imagens coletadas em situações como período noturno ou clima com neve podem ainda aumentar a diversidade e robustez dos testes.

Há também uma carência de bases com amostras de vagas em laterais de vias públicas. Um exemplo de base que contém imagens em vias e máscaras de segmentação é a COCO [Lin et al. 2014]. No entanto essa é uma base geral, com poucas amostras específicas para estacionamentos. Quanto a protocolos padronizados existem, por exemplo, os propostos em [de Almeida et al. 2015, Amato et al. 2017]. No entanto, esses protocolos tratam apenas dos problemas de classificação de vagas.

Pergunta de pesquisa: como propor protocolos de testes robustos para os problemas em aberto, e quais problemas existem nas bases de dados rotuladas atuais que precisam ser sanados a fim de tornar a execução dos protocolos viável e prática?

\section{Projetos em Andamento}

Atualmente, nossa equipe está trabalhando em novas bases e protocolos (Seção 3.6) e em métodos para a segmentação automatizada de vagas (Seção 3.2). Para as bases de dados, estamos trabalhando na rotulação por máscaras de segmentação de pelo menos $70 \%$ dos veículos das bases PKLot, CNRPARK-EXT e PLDs. Estamos também rotulando novas vagas de estacionamento nessas bases (especialmente na PLDs, que originalmente possui apenas caixas delimitadoras em veículos estacionados). Essas novas anotações podem contribuir para a criação e validação de métodos de segmentação de vagas, classificação de vagas, e métodos de detecção e segmentação de veículos e objetos em geral.

Os protocolos em desenvolvimento envolvem principalmente o uso de múltiplas bases (e.g. PKLot, CNRPark-EXT e PLds) com leave-one-out, onde uma base completa deve ser utilizada somente para os testes a cada iteração, a fim de evitar vieses. Métricas para os problemas de classificação e segmentação de vagas, como a Área Sob a Curva ROC (AUC-ROC), F1 e Precisão Média (AP) estão sendo estudadas para fazer parte do protocolo de testes, a fim de tornar os resultados de diferentes autores (que empregarem o protocolo) diretamente comparáveis. 
Já o método de segmentação de vagas sendo desenvolvido baseia-se na geração de mapas de calor dos estacionamentos, onde os mapas indicam onde os veículos geralmente param. Com esses mapas, que serão gerados através de redes neurais de segmentação semântica, será possível inferir as posições das vagas de estacionamento sem intervenção humana. A criação e testes desse método somente está sendo possível graças as extensões das bases que estão em andamento, como discutido anteriormente. Todos os resultados (bases e métodos) serão publicados e disponibilizados gratuitamente, como feito em nossos trabalhos anteriores [de Almeida et al. 2015, Almeida et al. 2018].

\section{Conclusão}

Nesse trabalho foram apresentados desafios ainda em aberto referentes a gerencia de vagas de estacionamento por câmera em cidades inteligentes, como a classificação de vagas (entre ocupadas e vazias) em ambientes sem amostras de treinamento; a segmentação automatizada de vagas; a criação de classificadores leves (para edge computing); a criação de métodos para detecção de vagas em vias públicas; e a confecção de novas bases de testes e protocolos. Métodos capazes de lidar com esses desafios podem melhorar a gerência dos sistemas de transporte de cidades inteligentes, guiando os motoristas (ou veículos autônomos) diretamente até a vaga mais próxima, economizando tempo e energia.

Foram apresentados ainda nossos projetos que estão em andamento. As extensões das bases que estão sendo desenvolvidas, e os novos protocolos de testes, almejam facilitar o desenvolvimento e comparação de novos métodos. O método de segmentação automática de vagas em desenvolvimento será um dos poucos disponíveis na literatura que apresentará resultados quantitativos em bases de testes de grande porte (serão utilizadas as extensões das bases e os protocolos sendo desenvolvidos para validar o método).

Esperamos que os desafios apresentados nesse trabalho, bem como os resultados dos projetos em andamento, sirvam de base e inspiração para futuros desenvolvimentos, que poderão tornar a vida dos cidadãos mais simples, e as cidades mais sustentáveis.

\section{Referências}

Agrawal, T. and Urolagin, S. (2020). Multi-angle parking detection system using mask r-cnn. In Proceedings of the 2020 BDET, volume 2020, pages 76-80. ACM.

Ahrnbom, M., Astrom, K., and Nilsson, M. (2016). Fast classification of empty and occupied parking spaces using integral channel features. In Proceedings of the IEEE CVPR Workshops, volume 2016, pages 1609-1615. IEEE.

Ahvenniemi, H., Huovila, A., Pinto-Seppä, I., and Airaksinen, M. (2017). What are the differences between sustainable and smart cities? Cities, 60:234-245.

Almeida, P. R., Oliveira, L. S., Britto, A. S., and Sabourin, R. (2018). Adapting dynamic classifier selection for concept drift. ESWA, 104:67-85.

Amato, G., Carrara, F., Falchi, F., Gennaro, C., Meghini, C., and Vairo, C. (2017). Deep learning for decentralized parking lot occupancy detection. ESWA, 72:327-334.

Bohush, R., Yarashevich, P., Ablameyko, S., and Kalganova, T. (2018). Extraction of image parking spaces in intelligent video surveillance systems. $M G V, 27(1-4): 47-62$.

Chen, L.-C., Sheu, R.-K., Peng, W.-Y., Wu, J.-H., and Tseng, C.-H. (2020). Video-based parking occupancy detection for smart control system. Applied Sciences, 10(3):1079. 
de Almeida, P. R., Oliveira, L. S., Britto, A. S., Silva, E. J., and Koerich, A. L. (2015). Pklot - a robust dataset for parking lot classification. ESWA, 42(11):4937 - 4949.

DESA, U. N. (2019). World urbanization prospects: The 2018 revision. United Nations: New York, NY, USA.

Djahel, S., Salehie, M., Tal, I., and Jamshidi, P. (2013). Adaptive traffic management for secure and efficient emergency services in smart cities. In 2013 IEEE PERCOM Workshops, pages 340-343. IEEE.

Hurst-Tarrab, N., Chang, L., Gonzalez-Mendoza, M., and Hernandez-Gress, N. (2020). Robust parking block segmentation from a surveillance camera perspective. Applied Sciences, 10(15):5364.

Jensen, T. H., Schmidt, H. T., Bodin, N. D., Nasrollahi, K., and Moeslund, T. B. (2017). Parking space occupancy verification-improving robustness using a convolutional neural network. In VISAPP, volume 6, pages 311-318. SCITEPRESS.

Koumetio Tekouabou, S. C., Abdellaoui Alaoui, E. A., Cherif, W., and Silkan, H. (2020). Improving parking availability prediction in smart cities with iot and ensemble-based model. Journal of King Saud University - Computer and Information Sciences.

Letaifa, S. B. (2015). How to strategize smart cities: Revealing the smart model. Journal of business research, 68(7):1414-1419.

Li, Z., Shahidehpour, M., Bahramirad, S., and Khodaei, A. (2017). Optimizing traffic signal settings in smart cities. IEEE Transactions on Smart Grid, 8(5):2382-2393.

Lin, T.-Y., Maire, M., Belongie, S., Hays, J., Perona, P., Ramanan, D., Dollár, P., and Zitnick, C. L. (2014). Microsoft coco: Common objects in context. In ECCV, pages 740-755. Springer.

Nagy, A. M. and Simon, V. (2018). Survey on traffic prediction in smart cities. Pervasive and Mobile Computing, 50:148-163.

Nieto, R. M., García-Martín, Á., Hauptmann, A. G., and Martínez, J. M. (2018). Automatic vacant parking places management system using multicamera vehicle detection. IEEE ITS, 20(3):1069-1080.

Nurullayev, S. and Lee, S.-W. (2019). Generalized parking occupancy analysis based on dilated convolutional neural network. Sensors, 19(2):277.

Padmasiri, H., Madurawe, R., Abeysinghe, C., and Meedeniya, D. (2020). Automated vehicle parking occupancy detection in real-time. In MERCon, pages 1-6. IEEE.

Satyanarayanan, M. (2017). The emergence of edge computing. Computer, 50(1):30-39.

Varghese, A. and Sreelekha, G. (2019). An efficient algorithm for detection of vacant spaces in delimited and non-delimited parking lots. IEEE ITS.

Vítek, S. and Melničuk, P. (2018). A distributed wireless camera system for the management of parking spaces. Sensors, 18(1):69.

Zhang, C. and Du, B. (2020). Image-based approach for parking-spot detection with occlusion handling. JTE, Part A: Systems, 146(9):04020098.

Zhang, W., Yan, J., and Yu, C. (2019). Smart parking system based on convolutional neural network models. In ICISCE, pages 561-566. IEEE, IEEE. 